\title{
Proximal Approach of Ultrasound-guided Suprascapular Nerve Block: Comparison with Subacromial Steroid Injection
}

\author{
Kyu Hwan Bae, Han Hoon Kim, Tae Kang Lim ${ }^{\square}$ \\ Department of Orthopedic Surgery, Nowon Eulji Medical Center, Eulji University School of Medicine, Seoul, Korea
}

Background: This study was undertaken to evaluate early clinical outcomes of ultrasound-guided suprascapular nerve block (SSNB) using a proximal approach, as compared with subacromial steroid injection (SA).

Methods: This retrospective study included a consecutive series of 40 patients of SSNB and 20 patients receiving SA, from August 2017 to August 2018. The visual analogue scale (VAS), American Shoulder Elbow Surgeon's score (ASES), University of California, Los Angeles score (UCLA), the 36 health survey questionnaire mental component summary (SF36-MCS), physical component summary (PCS), and range of motion (forward elevation, external rotation, and internal rotation) were assessed for clinical evaluations.

Results: Compared with the baseline, VAS, and ranges of motion in the SSNB group significantly improved at the 4-week follow-up (VAS scores improved from $6.7 \pm 1.6$ to $4.3 \pm 2.4, p<0.001$; all ranges of motion $p<0.05$ ), while other variables showed no statistically significant differences. All clinical variables were significantly improved in the SA group $(p<0.05)$. However, all clinical scores at the 4 -week follow-up showed no significant difference between groups.

Conclusions: Ultrasound-guided SSNB using proximal approach provides significant pain relief at 4-weeks after treatment, with statistically significant difference when compared with SA, suggesting that SSNB using proximal approach is a potentially useful option in managing shoulder pain. However, in the current study, it was less effective in improving shoulder function and health-related quality of life, compared with SA.

(Clin Shoulder Elbow 2019;22(4):210-215)

Key Words: Suprascapular nerve block; Subacromial injection; Ultrasonography; Shoulder; Pain management

\section{Introduction}

Suprascapular nerve block (SSNB) is commonly applied in patients with chronic shoulder pain, especially adhesive capsulitis, ${ }^{1)}$ recalcitrant shoulder pain, ${ }^{2)}$ and post-operative shoulder pain. ${ }^{3)}$ The suprascapular nerve is a branch of the brachial plexus from which it receives contributions from the $\mathrm{C} 5, \mathrm{C} 6$ and, more variably, C4 nerve roots. ${ }^{4)}$ The motor branch of the suprascapular nerve supplies to the supraspinatus and infraspinatus muscles. In addition, it is known to provide towards $70 \%$ sensation to the shoulder joint, acromioclavicular joint, coracohumeral ligament, coracoclavicular ligaments, and subacromial bursa. ${ }^{5,6)}$ The suprascapular nerve branches off the superior trunk of the brachial plexus, close to the anterior and posterior divisions, and runs through the supraclavicular fossa under the inferior belly of the omohyoid and trapezius muscles. It enters the supraspinous fossa under the superior transverse scapular ligament.

The classic ultrasound (US)-guided approach to block the suprascapular nerve is accomplished via a distal approach at the suprascapular notch where the suprascapular nerve enters the supraspinous fossa." However, the success of US-guided distal SSNB at the suprascapular notch is limited by the nerve's depth and inconsistent anatomic variation at the origin of the sensory branches. ${ }^{8)}$

Recently, after evaluating the feasibility in a cadaveric study, the proximal approach of SSNB was introduced which shows

Received October 30, 2019. Revised November 18, 2019. Accepted November 18, 2019.

Correspondence to: Tae Kang Lim

Department of Orthopedic Surgery, Nowon Eulji Medical Center, Eulji University, 68 Hangeulbiseok-ro, Nowoungu, Seoul 01830, Korea Tel: +82-2-970-8036, Fax: +82-2-973-3024, E-mail: fromspace@daum.net, ORCID: https://orcid.org/0000-0001-8752-3987 IRB approval: Nowon Eulji Medical Center, Eulji University (No. EMCIRB 19-105).

Financial support: None. Conflict of interests: None. 
accurate visualization of the suprascapular nerve. Before the suprascapular nerve passes through the suprascapular notch, the nerve could be identified more proximally and superficially under the omohyoid muscle in the supraclavicular region. The omohyoid muscle serves as a landmark to help identify and block the nerve with the ultrasound image. ${ }^{9)}$ However, current literature lacks data reporting clinical efficacy of the proximal approach in managing shoulder pain.

This study was therefore undertaken to evaluate early clinical outcomes of US-guided SSNB block using the proximal approach, and comparing with subacromial steroid (SA) injection. We hypothesized there would be no significant difference in clinical outcomes between groups at 4-weeks after treatment.

\section{Methods}

This retrospective study was performed after gaining approval from our institutional review board. From August 2017 to August 2018, patients who received ambulatory care for shoulder pain underwent US-guided SSNB or SA. From August 2017 to April 2018, consecutive series of patients underwent US-guided SSNB (SSNB group), and patients between March 2018 to August 2018 were administered US-guided SA injection group (SA group). Patients with adhesive capsulitis, rotator cuff tear, calcific tendinitis or postoperative pain were included in the study. All patients were evaluated with a thorough history, physical examination, and standard plain radiographs by the treating surgeon. The exclusion criteria for the study were (1) patients who underwent both SSNB and SA due to severe pain, and (2) patients who were lost to follow-up.

\section{Injection Procedures}

The 'proximally approached' SSNB was performed as follows: the patient was placed in the sitting position, with the shoulder in a neutral position and hand resting on the ipsilateral thigh. A high-frequency linear ultrasound transducer $(5-14 \mathrm{MHz}$ linear probe, Aplio 500; Canon Medical Systems, Tochigi, Japan) was applied to the side of the neck at the level of the cricoid cartilage in a transverse oblique plane to obtain the best view of the brachial plexus. We moved the transducer caudally to identify the departure of the suprascapular nerve from the superior trunk of the brachial plexus. The suprascapular nerve was traced to the maximum lateral position possible, changing the probe orientation gradually to an oblique sagittal plane. The inferior belly of the omohyoid muscle was identified as an additional ultrasonographic landmark. The suprascapular nerve was located beneath the omohyoid muscle. After disinfecting the lateral cutaneous area of the probe with betadine, $0.2 \%$ ropivacaine $(5 \mathrm{ml})$ was injected from lateral to medial using the in-plane technique (Fig. 1). During injection, we continuously monitored the suprascapular nerve to confirm that the injection was successfully injected just lateral to the nerve, and not inside. After administering the injection, we examined that the motor and sensory activities of the ipsilateral arm were intact.

SA injection was achieved as follows: The patient was kept in a sitting position with the arm behind the back (Crass position). ${ }^{10}$ A high-frequency linear ultrasound transducer $(5-14 \mathrm{MHz}$ linear probe, Aplio 500; Canon Medical Systems) was applied anterior to the acromion, and beside the coracoid process. The supraspinatus under the deltoid muscle was identified in the longitudinal sagittal plane. After disinfecting the superior cutaneous area of the probe with betadine, a $2 \mathrm{ml}$ mixture containing $1 \mathrm{ml}$ triam-
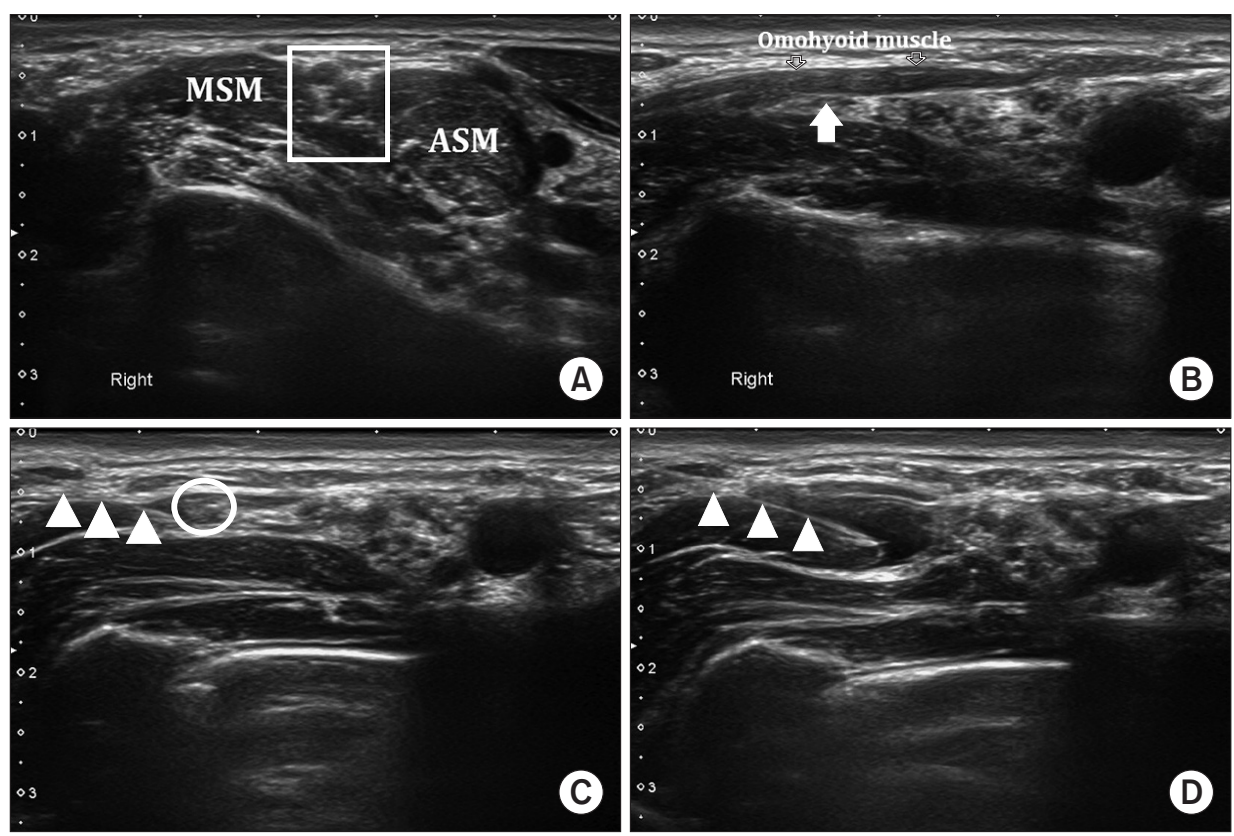

Fig. 1. Ultrasound image of proximally approached suprascapular nerve block. (A) C5, C6, C7 nerve roots are inside the quadrangle between middle scalene muscle (MSM) and anterior scalene muscle (ASM). (B) The suprascapular nerve branches off the superior trunk (arrow) of the brachial plexus just below the omohyoid muscle. (C) A needle is inserted from the lateral side of the probe with an in-plane technique until the needle tip reaches the suprascapular nerve. Triangle indicates the needle. Circle indicates the suprascapular nerve. (D) Ultrasound image after the suprascapular nerve block injection was done. Triangle indicates the needle. Injected fluid should ideally be around the suprascapular nerve, and not within. 
cinolone and $1 \%$ lidocaine $(1 \mathrm{ml})$ was injected from superior to inferior using the in-plane technique (Fig. 2). The target point of the injection was the bursal space between the supraspinatus and the deltoid muscle. During the injection, we continuously monitored the successful spread of the injected fluid just above the supraspinatus, and not within the tendon. After administration of the injection, we examined for intactness of the motor and sensory activities of the ipsilateral arm.

\section{Clinical Evaluation}

All patients were retrospectively evaluated using the information collected from medical histories, physical examination findings, injection records (including ultrasound images), and clinical scores obtained from electronic medical records. Demographic data, including age, sex, diagnosis of shoulder pathology, diabetes, thyroid disease, and trauma history, were obtained before administering the injection. For clinical evaluations, the visual analogue scale (VAS), American Shoulder Elbow Surgeon's score (ASES), University of California, Los Angeles score (UCLA), 36 health survey questionnaire mental component summary (SF36MCS), physical component summary (PCS), ${ }^{11,12)}$ and range of motion were evaluated before and at 4 weeks after injection for each group. Complications related to the injections were also investigated.

\section{Statistical Analysis}

Statistical analysis was carried out using the SPSS software ver. 15.0 (SPSS Inc., Chicago, IL, USA) as well as ver. 24.0 (IBM Corp., Armonk, NY, USA) for Windows. The total demographic data of patients were analyzed by an independent t-test for continuous variables, and the chi-squared test for nominal data. The paired t-test or Wilcoxon signed-rank test was used to compare

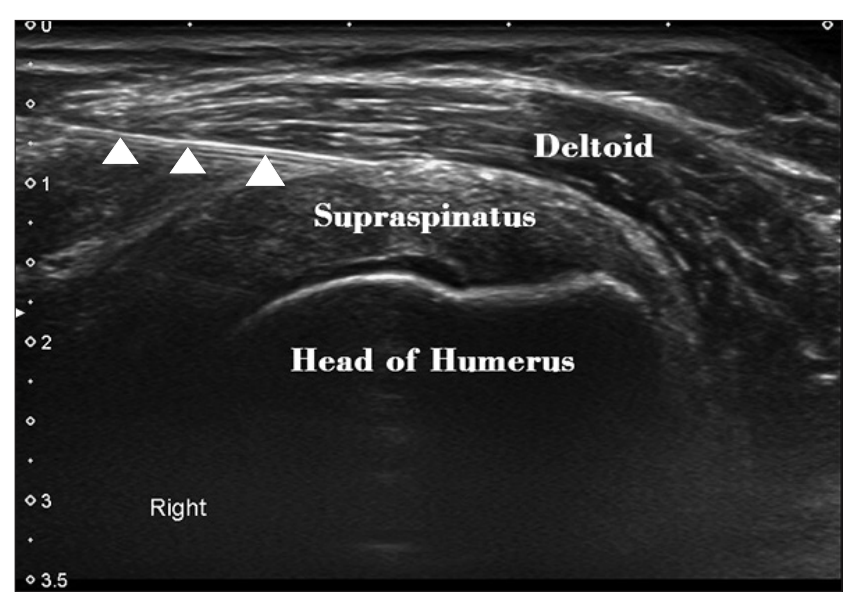

Fig. 2. Ultrasound image of subacromial steroid injection. A needle is inserted from superior to inferior with the in-plane technique until the needle tip reaches the subacromial space under the deltoid muscle. Triangle indicates the needle. the VAS, ASES, UCLA, SF36 scores, and range of motion, before and at 4 weeks after the injection. An independent t-test was applied for continuous variables and the chi-squared test for nominal data, to compare the final clinical results of the two groups. For all analyses, a p-value less than 0.05 is considered significant.

\section{Results}

From August 2017 to August 2018, a total of 74 consecutive patients underwent US-guided SSNB or SA. Of these, 5 patients who underwent both SSNB and SA injection due to severe pain, and 9 patients with loss of follow-up, were excluded. The remaining 60 patients (40 patients in SSNB group, 20 patients in SA group) were finally analyzed in this study.

The mean age of the SSNB group patients was $55.7 \pm 8.5$ years, and of the SA group patients was $56.9 \pm 11.6$ years. SSNB group included 26 females whereas SA group had 12 females. SSNB group and SA group included 8 and 3 patients, respectively, with diabetes mellitus, and 1 and 2 patients, respectively, with thyroid disease. Initial diagnosis for SSNB group included 15 patients with adhesive capsulitis, 8 patients with calcific tendinitis, 4 patients with a rotator cuff tear, and 13 patients with postoperative pain, while the SA group included 12 patients with adhesive capsulitis, 1 patient with calcific tendinitis, 4 patients with a rotator cuff tear, and 3 patients with postoperative pain. No significant differences were obtained for demographic data between both groups (Table 1).

\section{Clinical Outcome}

Clinical outcome after proximally approached SSNB by comparing baseline and at 4 weeks after injection was documented. The mean VAS, ASES, and UCLA scores show improvements after SSNB treatment: from baseline values $6.7 \pm 1.6,81.7 \pm$ 9.3 , and $24.6 \pm 6.3$, respectively, to $4.3 \pm 2.4,85.3 \pm 11.4$, and $25.7 \pm 6.3$, respectively. However, only VAS showed statistically significant difference $(p<0.001, p=0.055, p=0.274$, respectively; Fig. 3). Although SF36-MCS and SF36-PCS scores are improved, they were statistically not significant. Range of motion showed significant improvement for all three motions. Specific values are presented in Table 2.

Comparing the baseline and 4 weeks post-injection clinical outcomes after SA injection reveals statistically significant improvements in the mean VAS, ASES, and UCLA scores, from baseline $5.0 \pm 1.9,87.9 \pm 5.9$, and $26.8 \pm 4.7$, respectively, to $1.8 \pm 1.7,95.5 \pm 4.1$, and $32.1 \pm 2.2$, respectively, (all with $p<0.001)$. SF36-MCS and SF36-PCS scores also showed statistically significant improvements. Range of motion showed significant improvement at all three motions. Specific values are presented in Table 3.

Changes from baseline values of each variable in the clinical outcomes were documented. Changes in VAS, ASES, and UCLA 
Table 1. Demographic Data of the Two Groups

\begin{tabular}{|lccc}
\hline \multicolumn{1}{c}{ Variable } & $\begin{array}{c}\text { SSNB group } \\
(\mathrm{n}=40)\end{array}$ & $\begin{array}{c}\text { SA injection group } \\
(\mathrm{n}=20)\end{array}$ & p-value \\
\hline Age $(\mathrm{yr})$ & $55.7 \pm 8.5$ & $56.9 \pm 11.6$ & 0.664 \\
\hline Sex, male/female & $14 / 26$ & $8 / 12$ & 0.683 \\
\hline Diagnosis & & & 0.265 \\
\hline Frozen shoulder & $15(37.5)$ & $12(60.0)$ & \\
\hline Calcific tendinitis & $8(20.0)$ & $1(5.0)$ & \\
\hline Rotator cuff tear & $4(10.0)$ & $4(20.0)$ & 0.510 \\
\hline Postoperative pain & $13(32.5)$ & $3(15.0)$ & 0.179 \\
\hline Diabetes & $8(20.0)$ & $3(15.0)$ & 0.883 \\
\hline Thyroid & $1(2.5)$ & $2(10.0)$ & \\
\hline Trauma history & $9(22.5)$ & $5(25.0)$ & \\
\hline
\end{tabular}

Values are presented as mean \pm standard deviation, number only, or number (\%). SSNB: suprascapular nerve block, SA: subacromial steroid.

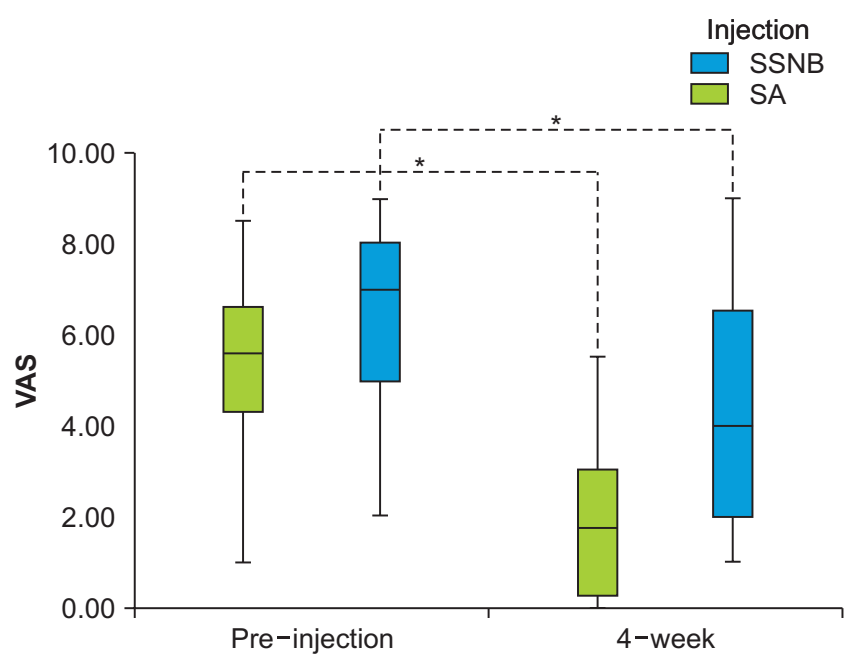

Fig. 3. Box-and-whisker plot displaying visual analogue scale (VAS) for subacromial steroid injection (SA) and suprascapular nerve blocks (SSNB) at pre-injection and 4-week after injection. VAS is presented as mean, standard deviation, and range.

*Statistically significant.

were greater in the SA group $(3.2 \pm 2.2,7.6 \pm 6.7$, and $5.3 \pm$ 4.8 , respectively) as compared to the SSNB group ( $2.4 \pm 2.1,3.5$ \pm 11.1 , and $1.1 \pm 6.4$, respectively); however, statistically significant difference was obtained only in the UCLA score $(p=0.009)$. Changes in the SF36-PCS score were statistically significant and greater in the SA group $(12.1 \pm 9.7)$ than SSNB group (10.2 \pm 15.9) $(p<0.001)$. Changes of range of motion were greater in $S A$ group than SSNB group in all three motions, but were statistically not significant. Specific values are presented in Table 4.

None of the enrolled patients had any complications (such as infection or hematoma) around the injected site. There was one patient with transient weakness on the upper extremity on the
Table 2. Clinical Outcome after Proximally Approached Suprascapular Nerve Block

\begin{tabular}{lccc}
\hline \multicolumn{1}{c}{ Variable } & Baseline & After 4-week & $p$-value \\
\hline Visual analogue scale & $6.7 \pm 1.6$ & $4.3 \pm 2.4$ & $<0.001$ \\
\hline $\begin{array}{c}\text { American Shoulder Elbow } \\
\text { Surgeon's score }\end{array}$ & $81.7 \pm 9.3$ & $85.3 \pm 11.4$ & 0.055 \\
\hline $\begin{array}{l}\text { University of California, } \\
\text { Los Angeles score }\end{array}$ & $24.6 \pm 6.3$ & $25.7 \pm 6.3$ & 0.274 \\
$\begin{array}{l}\text { SF36-mental component } \\
\text { summary }\end{array}$ & $69.3 \pm 14.2$ & $71.5 \pm 14.8$ & 0.349 \\
\hline $\begin{array}{c}\text { SF36-physical component } \\
\text { summary }\end{array}$ & $65.5 \pm 14.4$ & $75.8 \pm 55.2$ & 0.290 \\
\hline Range of motion $\left(^{\circ}\right)$ & $122.0 \pm 35.0$ & $137.4 \pm 33.2$ & $<0.001$ \\
\hline Forward elevation & $54.7 \pm 24.0$ & $64.0 \pm 21.2$ & $<0.001$ \\
\hline External rotation side & L1.9 \pm 3.8 & T12.7 \pm 4.0 & 0.001 \\
\hline Internal rotation back & & & \\
\hline
\end{tabular}

Values are presented as mean \pm standard deviation.

SF36: 36 health survey questionnaire.

Table 3. Clinical Outcome after Sub-acromial Steroid Injection

\begin{tabular}{|c|c|c|c|}
\hline Variable & Baseline & After 4-week & $p$-value \\
\hline Visual analogue scale & $5.0 \pm 1.9$ & $1.8 \pm 1.7$ & $<0.001$ \\
\hline $\begin{array}{l}\text { American Shoulder Elbow } \\
\text { Surgeon's score }\end{array}$ & $87.9 \pm 5.9$ & $95.5 \pm 4.1$ & $<0.001$ \\
\hline $\begin{array}{l}\text { University of California, } \\
\text { Los Angeles score }\end{array}$ & $26.8 \pm 4.7$ & $32.1 \pm 2.2$ & $<0.001$ \\
\hline $\begin{array}{l}\text { SF36-mental component } \\
\text { summary }\end{array}$ & $71.1 \pm 9.8$ & $77.7 \pm 6.5$ & 0.021 \\
\hline $\begin{array}{l}\text { SF36-physical component } \\
\text { summary }\end{array}$ & $64.8 \pm 10.5$ & $76.9 \pm 7.5$ & $<0.001$ \\
\hline \multicolumn{4}{|l|}{ Range of motion $\left(^{\circ}\right)$} \\
\hline Forward elevation & $126.1 \pm 33.8$ & $155.8 \pm 26.1$ & 0.001 \\
\hline External rotation side & $58.9 \pm 22.2$ & $76.4 \pm 14.3$ & 0.002 \\
\hline Internal rotation back & $\mathrm{L} 1.8 \pm 3.9$ & $\mathrm{~T} 11.2 \pm 4.6$ & 0.001 \\
\hline
\end{tabular}

Values are presented as mean \pm standard deviation.

SF36: 36 health survey questionnaire.

day of injection in SSNB group.

\section{Discussion}

Results of this study reveal that application of SSNB using a proximal approach provides significant improvement in pain VAS at 4-weeks after treatment, and the mean change of pain VAS from the baseline score is comparable to SA injection. These results indicate that SSNB using proximal approach is a potential option in managing shoulder pain. Although all clinical variables examined at 4-week follow-up visit were not signifi- 
Table 4. Changes of Each Clinical Outcome from the Baseline

\begin{tabular}{lccc}
\hline \multicolumn{1}{c}{ Delta $(\Delta)$ value } & SSNB & SA injection & $p$-value \\
\hline Visual analogue scale & $2.4 \pm 2.1$ & $3.2 \pm 2.2$ & 0.232 \\
$\begin{array}{l}\text { American Shoulder Elbow } \\
\text { Surgeon's score }\end{array}$ & $3.5 \pm 11.1$ & $7.6 \pm 6.7$ & 0.096 \\
\hline $\begin{array}{l}\text { University of California, } \\
\text { Los Angeles score }\end{array}$ & $1.1 \pm 6.4$ & $5.3 \pm 4.8$ & 0.009 \\
$\begin{array}{l}\text { SF36-mental component } \\
\text { summary }\end{array}$ & $2.2 \pm 14.2$ & $6.5 \pm 10.9$ & 0.209 \\
\hline $\begin{array}{l}\text { SF36-physical component } \\
\text { summary }\end{array}$ & $10.2 \pm 15.9$ & $12.1 \pm 9.7$ & $<0.001$ \\
Range of motion $\left({ }^{\circ}\right)$ & $15.5 \pm 20.4$ & $29.7 \pm 30.3$ & 0.082 \\
\hline Forward elevation & $9.2 \pm 12.2$ & $17.5 \pm 19.7$ & 0.114 \\
$\quad$ External rotation side & $1.2 \pm 2.0$ & $2.6 \pm 2.9$ & 0.070 \\
\hline Internal rotation back & & & \\
\hline
\end{tabular}

Values are presented as mean \pm standard deviation.

SSNB: suprascapular nerve block, SA: subacromial steroid, SF36: 36 health survey questionnaire.

cantly different between groups, improvement from the baseline levels in UCLA and SF36-PCS scores were more pronounced in the SA group than the SSNB group, thereby indicating that SSNB injection would be less effective in improving shoulder function and health-related quality of life than SA injection.

The proximal approach to the suprascapular nerve has recently been introduced. Siegenthaler et al. ${ }^{9)}$ described the difficulties in visualizing the suprascapular nerve with the conventional distal approach, and demonstrated the proximal approach of SSNB under US guidance in cadavers. Later, Hackworth ${ }^{13 \text { ) }}$ demonstrated a successful block of suprascapular nerve after it exits the superior trunk. One cadaveric study also showed that in-plane US-guided needle injection with a $5 \mathrm{ml}$ volume for SSNB successfully stained the suprascapular nerve in $90 \%$ of cases, supporting the technical ease and reliability of this approach.

Despite the usefulness proven in cadaveric studies, clinical outcomes of SSNB using the proximal approach have rarely been reported. Ours is the first study that evaluates the effect of the SSNB technique in managing shoulder pain, compared to SA injection. The suprascapular nerve was easily identified under US guidance in all cases. Two important technical tips to identify the nerve must be noted: first, the suprascapular nerve was identified as leaving the superior trunk of the brachial plexus, following which it travels in the intermediate space between the investing superficial cervical fascia and the prevertebral fascia; second, the omohyoid muscle is another landmark to identify the nerve. The nerve runs first inferior, and then parallel to the inferior belly of the omohyoid, subsequently passing posteriorly through the inferior region of the posterior triangle, toward the superior aspect of the scapula. Using this approach, the accuracy of identifying the nerve and injecting the blockade needs to be improved. However, the study showed only modest improvement of the VAS score, with no improvement in other parameters of functional scores or health-related quality of life at 4-weeks of injections. A recent systematic review demonstrated that SSNB is statistically, but not clinically, superior to control for the area under curve of pain VAS score with a mean difference of $1.01 \mathrm{~cm}$, suggesting that SSNB produced only modest improvements in analgesic outcomes. ${ }^{14)}$ Although this finding is similar to our study, we found that the proximal approach was superior in terms of identifying the nerve and the use of US guidance, thereby obviously improving the accuracy, as compared to SSNB using landmark-guided (blind) posterior approach. However, it remains unknown why this improved accuracy could not be translated to improved clinical outcomes in this study.

Unfortunately, this study also failed to show the superiority of SSNB using proximal approach over administration of steroid injections.

SA injections are known to be effective as intra-articular steroid injections in reducing pain. ${ }^{15)}$ The chronic inflammation process of common shoulder disease, especially adhesive capsulitis, influences functioning of the shoulder joint. It is a well-known fact that corticosteroids exert properties of antiinflammation. Therefore, the use of corticosteroids is effective for improving shoulder function, especially adhesive capsulitis. ${ }^{16)}$ It was predictable that the steroid injection group shows a good clinical outcome; however, we endeavored to compare this technique with the new proximally approached SSNB. This will be helpful for treating patients unable to receive steroid injection because of diabetes. ${ }^{17)}$ Since steroids influence the blood sugar level, current evidence suggests that physiotherapy could be an alternative. ${ }^{18)}$ In our opinion, SSNB can be an alternative to steroid injection, especially for patients with severe pain.

Coory et al. ${ }^{19)}$ demonstrated that SSNB resulted in better pain and functional results than SA for symptomatic rotator cuff tears. However, Coory et al. ${ }^{19)}$ performed a distal approach targeting the suprascapular nerve at the suprascapular notch. For advantage of ease and accuracy, the proximal approach SSNB has some merits, but further studies are required to completely elucidate the procedure.

\section{Limitation}

Several limitations of this study need to be mentioned. First, this study was retrospectively designed and had a short followup term of only 4 weeks. Second, since comparison with conventional distally approached SSNB might be a better study design, future work is required to compare the two techniques as a prospective study design. Third, this study includes varying conditions of shoulder pain, such as frozen shoulder, rotator cuff tear, calcific tendinitis and postoperative shoulders. This heterogeneity of study cohort with small sample size is an important limitation, and requires sub-analysis for each disease group. 


\section{Conclusion}

US-guided SSNB using the proximal approach provides significant pain relief at 4-week after treatment, but without statistically significant difference when compared with SA injection. Our results indicate that SSNB using the proximal approach is a useful option in managing shoulder pain. However, in this study, it was less effective in improving shoulder function and healthrelated quality of life, compared with SA injection.

\section{References}

1. Dahan TH, Fortin L, Pelletier M, Petit M, Vadeboncoeur R, Suissa S. Double blind randomized clinical trial examining the efficacy of bupivacaine suprascapular nerve blocks in frozen shoulder. J Rheumatol. 2000;27(6):1464-9.

2. Chang KV, Hung CY, Wang TG, Yang RS, Sun WZ, Lin CP. Ultrasound-guided proximal suprascapular nerve block with radiofrequency lesioning for patients with malignancyassociated recalcitrant shoulder pain. J Ultrasound Med. 2015;34(11):2099-105. doi: 10.7863/ultra.14.12042.

3. Park JY, Bang JY, Oh KS. Blind suprascapular and axillary nerve block for post-operative pain in arthroscopic rotator cuff surgery. Knee Surg Sports Traumatol Arthrosc. 2016;24(12):387783. doi: 10.1007/s00167-015-3902-3.

4. Ajmani ML. The cutaneous branch of the human suprascapular nerve. J Anat. 1994;185(Pt 2):439-42.

5. Ritchie ED, Tong D, Chung F, Norris AM, Miniaci A, Vairavanathan SD. Suprascapular nerve block for postoperative pain relief in arthroscopic shoulder surgery: a new modality? Anesth Analg. 1997;84(6):1306-12. doi: 10.1097/00000539199706000-00024.

6. Vorster W, Lange CP, Briët RJ, et al. The sensory branch distribution of the suprascapular nerve: an anatomic study. J Shoulder Elbow Surg. 2008;17(3):500-2. doi: 10.1016/ j.jse.2007.10.008.

7. Harmon D, Hearty C. Ultrasound-guided suprascapular nerve block technique. Pain Physician. 2007;10(6):743-6.

8. Blasco L, Laumonerie P, Tibbo M, et al. Ultrasound-guided proximal and distal suprascapular nerve blocks: a comparative cadaveric study. Pain Med. In press, available on 24 July 2019. doi: 10.1093/pm/pnz157.

9. Siegenthaler A, Moriggl B, Mlekusch S, et al. Ultrasound-guided suprascapular nerve block, description of a novel supracla- vicular approach. Reg Anesth Pain Med. 2012;37(3):325-8. doi: 10.1097/AAP.0b013e3182409168.

10. Shah NP, Miller TT, Stock H, Adler RS. Sonography of supraspinatus tendon abnormalities in the neutral versus Crass and modified Crass positions: a prospective study. J Ultrasound Med. 2012;31(8):1203-8. doi: 10.7863/jum.2012.31.8.1203.

11. Laucis NC, Hays RD, Bhattacharyya T. Scoring the SF36 in orthopaedics: a brief guide. J Bone Joint Surg Am. 2015;97(19):1628-34. doi: 10.2106/JBJS.O.00030.

12. Brazier JE, Harper R, Jones NM, et al. Validating the SF-36 health survey questionnaire: new outcome measure for primary care. BMJ. 1992;305(6846):160-4. doi: 10.1136/bmj.305. 6846.160.

13. Hackworth RJ. A new and simplified approach to target the suprascapular nerve with ultrasound. J Clin Anesth. 2013;25(4):347-8. doi: 10.1016/j.jclinane.2013.01.006.

14. Cho N, Kang RS, McCartney CJL, et al. Analgesic benefits and clinical role of the posterior suprascapular nerve block in shoulder surgery: a systematic review, meta-analysis and trial sequential analysis. Anaesthesia. In press, available on 3 October 2019. doi: 10.1111/anae.14858.

15. Goyal T, Singh A, Negi P, Kharkwal B. Comparative functional outcomes of patients with adhesive capsulitis receiving intraarticular versus sub-acromial steroid injections: case-control study. Musculoskelet Surg. 2019;103(1):31-5. doi: 10.1007/ s12306-018-0538-8.

16. Xiao RC, Walley KC, DeAngelis JP, Ramappa AJ. Corticosteroid injections for adhesive capsulitis: a review. Clin J Sport Med. 2017;27(3):308-20. doi: 10.1097/JSM.0000000000000358.

17. Choudhry MN, Malik RA, Charalambous CP. Blood glucose levels following intra-articular steroid injections in patients with diabetes: a systematic review. JBJS Rev. 2016;4(3):01874474201603000-00002. doi: 10.2106/JBJS.RWW.O.00029.

18. Sun Y, Lu S, Zhang P, Wang Z, Chen J. Steroid injection versus physiotherapy for patients with adhesive capsulitis of the shoulder: a PRIMSA systematic review and metaanalysis of randomized controlled trials. Medicine (Baltimore). 2016;95(20):e3469. doi: 10.1097/MD.0000000000003469.

19. Coory JA, Parr AF, Wilkinson MP, Gupta A. Efficacy of suprascapular nerve block compared with subacromial injection: a randomized controlled trial in patients with rotator cuff tears. J Shoulder Elbow Surg. 2019;28(3):430-6. doi: 10.1016/ j.jse.2018.11.051. 